This is the peer reviewed version of the following article:

Sandiumenge F., Bagués N., Santiso J., Paradinas M., Pomar A., Konstantinovic Z., Ocal C., Balcells L., Casanove M.-J., Martínez B.. Misfit Dislocation Guided Topographic and Conduction Patterning in Complex Oxide Epitaxial Thin Films. Advanced Materials Interfaces, (2016). 3. 1600106: - . 10.1002/admi.201600106,

which has been published in final form at https://dx.doi.org/10.1002/admi.201600106. This article may be used for non-commercial purposes in accordance with Wiley Terms and Conditions for Use of Self-Archived Versions. 


\section{Misfit Dislocation Guided Topographic and Conduction Patterning in Complex Oxide Epitaxial Thin Films}

Felip Sandiumenge, * Núria Bagués, José Santiso, Markos Paradinas, Alberto Pomar, Zorica Konstantinovic, Carmen Ocal, Lluís Balcells, Marie-Jo Casanove, and Benjamín Martínez

Dr. F. Sandiumenge, N. Bagués, Dr. A. Pomar, Dr. Z. Konstantinovic, Prof. C. Ocal, Dr. Ll. Balcells, Prof. B. Martínez

ICMAB-CSIC, Campus de la UAB, 08193 Bellaterra, Spain

E-mail: felip@icmab.es

N. Bagués, J. Santiso, M. Paradinas

CIN2 (CSIC-ICN), Campus de la UAB, 08193 Bellaterra, Spain

Prof. M. -J. Casanove

CNRS, CEMES, Universite de Toulouse UPS, 29 Rue J. Marvig, Toulouse 31055, France

Keywords: complex oxides, misfit dislocations, strain, surface patterning, surface current

Interfacial dissimilarity has emerged in recent years as the cornerstone of emergent interfacial phenomena, while enabling the control of electrical transport and magnetic behavior of complex oxide epitaxial films. As a step further towards the lateral miniaturization of functional nanostructures, this work uncovers the role of misfit dislocations in creating periodic surface strain patterns that can be efficiently used to control the spatial modulation of mass transport phenomena and bandwidth dependent properties on a $\sim 20 \mathrm{~nm}$ length scale. The spontaneous formation of surface strain-relief patterns in $\mathrm{La}_{0.7} \mathrm{Sr}_{0.3} \mathrm{MnO}_{3} / \mathrm{LaAlO}_{3}$ films results in lateral periodic modulations of the surface chemical potential and tetragonal distortion, controlling the spatial distribution of preferential nucleation sites and the bandwidth of the 
epilayer, respectively. These results provide insights into the spontaneous formation of straindriven ordered surface patterns, topographic and functional, during the growth of complex oxide heterostructures on lengths scales far below the limits achievable through top-down approaches.

\section{Introduction}

Thin film heteroepitaxy of complex oxides has evolved in recent years to a fascinating platform for the manipulation of materials properties by altering the subtle energy landscape of competing interactions through epitaxial strain and dissimilarity. ${ }^{[1,2]}$ Notably, this strategy has led to the discovery of exotic interfacial phenomena, ${ }^{[3,4]}$ while opening the possibility to tune the bulk transport, magnetic, ferroelectric, and multiferroic properties of thin films. ${ }^{[5-9]}$ However, next generation nanodevices demand a further step towards miniaturization, facing challenging strategies to controllably manipulate the lateral modulation of atomic length scales. In semiconductor epitaxy, this goal can be achieved through the Stranski-Krastanov growth mode, leading to the formation of self-assembled quantum dots on a wetting layer ${ }^{[10]}$ driven by lateral gradients in the surface chemical potential. ${ }^{[11]}$ This strategy, however, typically leads to nanostructures exhibiting broad size distributions and poor positional order. Strained films, on the other hand, usually relax by misfit dislocations (MDs) above a critical thickness at which their elastic energy exceeds the energy of the interfacial dislocation network. ${ }^{[12]}$ The overlapping of strain fields emanating from individual dislocations causes lateral modulations of lattice distortions which may extent up to the free surface. Therefore, highly organized MD networks buried at the substrate-film interface not only modulate the physical properties of thin films, but in addition can promote the growth of ordered nanostructures on their surfaces. In this sense, MDs have been used to produce strain guided patterned surfaces in semiconductor ${ }^{[13,14]}$ and metal ${ }^{[15,16]}$ systems, and more recently to tune 
Dirac surface sates in topological insulators. ${ }^{[17]}$ However, to date, the extension of this concept to oxide epitaxy remains elusive.

A unique property of dislocations, that make them highly appealing for creating new functional nanostructures, is their multiscale character. While being essentially linear defects, they store their elastic energy at comparatively large distances (several nanometers) from their sub-nanometer core. As a consequence, they can modify the properties of the host material in two different length scales. On the one hand, dislocation lines can be considered as a separate phase exhibiting its own physical behavior. ${ }^{[18]}$ A clear manifestation of their singularity, for instance, comes from the observation that oxygen deficient dislocations in STO ${ }^{[19-21]}$ exhibit bistable resistive switching, ${ }^{[22]}$ or that their non-superconducting cores induce pinning of the magnetic flux lines in type-II superconductors. ${ }^{[23,24]}$ On the other hand, owing to the strong sensitivity of the electronic structure to strain, MD long range strain fields are key for the realization of periodic functional (magnetic, electronic or catalytic ${ }^{[25]}$ ) bulk and surface patterns. Particularly, in manganite thin films, theoretical studies show that the sign of the misfit strain profoundly alters their magnetotransport response. ${ }^{[6]}$ A compressive/tensile biaxial strain promotes a stronger/weaker Mn $3 d-\mathrm{O} 2 p$ orbital overlapping leading to a broader/narrower bandwidth, allowing a local control on the electrical conduction by imposing spatial strain modulations. As MDs typically form square networks, they provide positional order along two mutually perpendicular directions for the creation of self-organized surface templates, ${ }^{[13-17]}$ in contrast with substrate steps which only induce unidirectional ordering. ${ }^{[26]}$ Notably, dislocation spacings within the $10-20 \mathrm{~nm}$ range are commonly obtained, which is far below the miniaturization limits achievable in oxides by top-down approaches, ${ }^{[27]}$ and can be manipulated through an appropriate choice of the substrate and the thickness of the film. Despite the strong potential of MDs in oxide heterostructures to create ordered functional patterns, however, their associated long range strains remain poorly understood. In particular, previous studies have been mainly focused on their detrimental 
effect on the spontaneous polarization in ferroelectric materials. ${ }^{[28-31]}$ This circumstance can be understood by considering the difficulty to predict the misfit relaxation behavior of the framework structures building these materials, as well as to the challenging requirement of correlating local surface properties with localized buried strain sources.

In this work we address these issues using thin films of the highly spin-polarized room temperature $\left(\mathrm{T}_{\mathrm{C}} \sim 370 \mathrm{~K}\right)$ half-metal ferromagnet, $\mathrm{La}_{0.7} \mathrm{Sr}_{0.3} \mathrm{MnO}_{3}(\mathrm{LSMO}),{ }^{[32]}$ as a model system. The interest on strategies to functionalize their surfaces stems from its strong potential in technological applications such as tunneling junctions and spin filters, ${ }^{[33]}$ high density data storage media, ${ }^{[34]}$ nanosensors or catalysts. ${ }^{[35]}$ It is shown that strain modulated thin films constitute an ideal scenario to investigate the complex interplay between strain and functionality at a local level, while providing a clue for the realization of spontaneous functional nanostructures in the $\sim 20 \mathrm{~nm}$ range.

\section{Results and discussion}

\subsection{Dislocation structure and strain evolution}

Figure 1a shows a cross section HRTEM image of the 2nm thick film, exhibiting coherent, dislocation free, interfaces and flat surfaces. The out-of-plane lattice parameter determined by HR-XRD is 3.98(1) $\AA$, which for a fully strained film yields a Poisson's ratio $v=\varepsilon_{\mathrm{zz}} /\left(\varepsilon_{\mathrm{zz}}\right.$ $\left.2 \varepsilon_{x x}\right)=0.35$, very close to that derived for tensile strained LSMO/STO films in their elastic regime, $v=0.33 .{ }^{[7]}$ As shown in Figure $1 b$, the $3.5 \mathrm{~nm}$ thick film, on the other hand, exhibits an undulated surface and a dislocated interface (see the encircled MD). Careful inspection of this image shows that the surface undulations are due to both, a donwards bending of the horizontal atomic rows above the dislocation core (see also Figure 1c), and outgrowths, as indicated by arrowheads, that as a general trend form on the surface at each side of the 
projected line of the buried MDs, $\sim 4 \mathrm{~nm}$ away from them (see Figure 1b). In thicker films, the outgrowths are no longer present, while the bending of atomic planes prevails independently of film thickness, Figure S1.

An enlarged view of the core structure of the encircled MD is presented in Figure 1c. The Burgers circuit yields a Burgers vector $\mathbf{b}_{\mathrm{x}}=[100]$, parallel to the interface, as indicated in the Figure. The two arrowed vertical atomic rows terminating at the slip plane bare witness of an split core: $[100] \rightarrow 1 / 2[100]+1 / 2[100]\left(\mathbf{b}_{\mathrm{x}}=\mathbf{b}_{1}+\mathbf{b}_{2}\right)$. A similar core structure has been recently identified as a polymorphic form of $\mathbf{b}_{\mathrm{x}}=[100]$-type dislocations in $\mathrm{MgO} \cdot{ }^{[36]}$ In fact, analysis of several MD cores indicated that this core splitting is a general trend among the observed MDs. The two adjacent $\mathbf{b} 1$ and $\mathbf{b} 2$ partial dislocations build a rather compact core structure. The displacement field above the core is clearly manifested as a downwards bending of the atomic rows, with a maximum amplitude of $\sim 1.25 \AA$ at the horizontal locus of the buried dislocation. Figure 1d shows maps of the in-plane, $\varepsilon_{x x}(x, z)$, and out-of-plane, $\varepsilon_{\mathrm{zz}}(x, z)$, strain components around the MD, computed from the experimental image shown in (c) using Geometrical Phase Analysis. ${ }^{[37]}$ The positions of the two partials in the composite core are clearly discerned, along with the compressive (red, yellow) and tensile regions (magenta, blue) extending into the substrate and the film, respectively. It can be observed that the misfit relieving tensile strains, $\varepsilon_{x x}$, draw two arms propagating up to the film surface, whilst normal strains, $\varepsilon_{z z}$, rapidly vanish as a result of the boundary condition of a free (001) surface, $\sigma_{z z}=\sigma_{z x}=\sigma_{y x}=0$ ( $\sigma_{i i}$ are components of the stress tensor $)$.

A projection of the MD network on the interface plane obtained by orientation contrast SEM is shown Figure 2a. The MD network is formed by irregularly spaced short ( 100 nm) dislocation segments along the [100] and [010] directions, with average spacing $\langle S\rangle=$ $25 \pm 10 \mathrm{~nm}$ (see Figure S1). These lines correspond to the projection of half-loops onto the interfacial plane, where they leave a MD segment which increases in length as the threading 
segments move apart. With increasing film thickness to $6 \mathrm{~nm},\langle\mathrm{~S}\rangle$ reduces to $19 \pm 6 \mathrm{~nm}$, while most of dislocations run across the limits of the imaged $1000 \mathrm{~nm} \times 1000 \mathrm{~nm}$ areas (see Figure $\mathrm{S} 1)$. The theoretical dislocation spacing for full relaxation, $b_{x} / \varepsilon=16.5 \mathrm{~nm}$, is achieved for the $14 \mathrm{~nm}$ thick film, with $\langle S\rangle=16 \pm 3 \mathrm{~nm}$. According to equilibrium theory, the energy barrier needed for a half-loop of critical radius to survive increases with decreasing misfit strain. ${ }^{[38]}$ Therefore, as the misfit is relieved by expansion of existing half-loops, the nucleation of new ones becomes kinetically suppressed. As a consequence, the average dislocation length increases whilst their lateral spacing narrows as the films thicken, in excellent agreement with experimental observations (see Figure S1). Since the glide plane coincides with the interface plane, the MDs can easily rearrange their positions on that plane to minimize their elastic interactions, resulting in increasingly ordered patterns.

In order to analyze the morphological transition occurring between 2 and $3.5 \mathrm{~nm}$, Figure 2 compares the MD network shown in (a) with a topographic AFM image of the same film (b). In agreement with the cross section HRTEM image shown in Figure 1b, it is clearly seen that the surface topography consists of a network of ridges and terraces mimicking the underlying dislocation network. Hence, the outgrowths shown in Figure $1 \mathrm{~b}$ correspond to a section of the ridges observed in the AFM image. To visualize the bulk strain state of the dislocated films, we obtained planar view strain sensitive LAADF images from the 6nm thick film (see Figure 3a and $\mathrm{b}$ ) and compared them with the spatial distribution of the residual inplane strain $\varepsilon_{x x}(x, y)+\varepsilon_{0},\left(\varepsilon_{0}=-2.3 \%\right.$ is the background misfit strain), arising from a square network of $\mathbf{b}_{\mathrm{x}}=[100]$ and $\mathbf{b}_{\mathrm{y}}=[010]$ dislocations, using continuum isotropic elasticity (see Figure 3c). We employed the isotropic-average shear modulus of LSMO derived from the Voigt-Reuss-Hill averaging, ${ }^{[39]}$ using the cubic elastic stiffness coefficients reported by Darling et al. ${ }^{[40]} G=50.98 \mathrm{GPa}$. Note that the LSMO is softer than the LAO substrate $(G=$ 133.10 $\left.\mathrm{GPa}^{[41]}\right)$. In this situation, the core of the dislocation is predicted to lie in the film 
very close to the interface, ${ }^{[42]}$ in agreement with the present observations. The calculated strain map shows good match with the LAADF experimental image, in which darker areas correspond to a lower degree of misfit relaxation between dislocations. Minimum relaxation levels are also attained along the locus of dislocation lines and, to a lower extent, at the crossing points, as also resolved in the experimental image [see Figure 3b]. This suggests that the observed topography results from preferential growth at surface sites with a minimum level of residual strain.

Since here we are interested on the effect of strains on surface phenomena, to confirm this hypothesis, here we estimate the strain state of the free film surface using displacement fields derived from the image dislocation approach. ${ }^{[39]}$ The vertical and horizontal atomic displacements, $u_{\mathrm{z}}$ and $u_{\mathrm{x}}$, at the free surface of a film with thickness $d$, induced by an interfacial dislocation with Burgers vector $b_{x}$, are given by: ${ }^{[43]}$

$$
\begin{aligned}
& u_{z}(x)=\frac{b_{x}}{\pi}\left(\frac{d^{2}}{x^{2}+d^{2}}\right) \\
& u_{x}(x)=\frac{b_{x}}{\pi}\left[\frac{-d x}{x^{2}+d^{2}}+\tan ^{-1}\left(\frac{x}{d}\right)\right]
\end{aligned}
$$

From Equation 1a, the amplitude of the downwards atomic displacement at the dislocation coordinate $x=0$ is $b_{x} / \pi \sim 1.20 \AA\left(b_{x}=a_{\mathrm{LSMO}}=3.79 \AA\right)$, in excellent agreement with the displacement field determined from Figure 1c. The modulation of the surface strain is then obtained by differentiation of Equation $1 \mathrm{~b}, \varepsilon_{x x}(x)=\partial u_{x}(x) / \partial x$,

$$
\varepsilon_{x x}(x)=\frac{b_{x} d}{\pi\left(x^{2}+d^{2}\right)}\left(\frac{x^{2}-d^{2}}{x^{2}+d^{2}}+1\right)
$$


The tensile strain given by Equation 2 is superimposed over the background compressive misfit strain due to the substrate, $\varepsilon_{0}$, yielding a residual surface strain, $\varepsilon_{x x}(x)+\varepsilon_{0}$, as plotted in Figure $4 \mathrm{a}$ for the 3.5 and $6 \mathrm{~nm}$ thick films, respectively. The Figure includes three dislocations, the central one at $x=0$. For the $3.5 \mathrm{~nm}$ thick film, assuming a dislocation spacing of $25 \mathrm{~nm}$, almost total relaxation $\left(\varepsilon_{x x}(x)+\varepsilon_{0}\right) \sim 0$ occurs at each side of the dislocation line at a distance of about $4 \mathrm{~nm}$. For the $6 \mathrm{~nm}$ thick one, assuming a dislocation spacing of $19 \mathrm{~nm}$, the overlapping of strain fields erases the fluctuation between adjacent MDs. In this case complete relaxation is achieved within a region of size similar to the thickness of the film located between adjacent dislocations. However, for those two thicknesses, specially for the thinner film, the dispersion of $S$ values is still high enough to expect the coexistence of both types of modulation in the same sample.

\subsection{Evolution of film topography}

The link between the morphological evolution of a free surface and its strain state is given by the surface chemical potential $\mu(x)=\mu_{0}+\gamma \Omega \kappa(x)+\Omega w(x),{ }^{[11]}$ where $\mu_{0}$ is the chemical potential of the unstressed flat surface, $\gamma$ is the surface free energy per unit area, $\Omega$ is the volume of a growth unit, $\kappa(x)$ is the surface curvature and $w(x)$ is the local surface strain energy density. ${ }^{[44]}$ Assuming a flat surface, $\kappa \sim 0$, the modulation of the driving force for strain induced surface mass transport due to an underlying pure edge MD located at $x=0, \Delta \mu_{0}(x)=$ $\mu(x)-\mu_{0}=\Omega w(x)$, can be expressed as:

$$
\Delta \mu_{0}(x)=\Omega M\left(\varepsilon_{0}^{2}+\varepsilon_{0} \varepsilon_{x x}(x)+\frac{\varepsilon_{x x}^{2}(x)}{2+2 v}\right)
$$


where $M=2 G(1+v) /(1-v)$. According to Equation 3, growth units will migrate from highly strained regions to find stable positions at locations exhibiting minimum $\Delta \mu_{0}(x)$ values at each side of the dislocation. To illustrate this, Figure $4 \mathrm{~b}$ depicts the chemical potential reduction relative to the position of the MD, as a function of distance, $x: \Delta \mu_{d}(x)=\mu(x)-\mu_{d}\left(\mu_{d}\right.$ is the surface chemical potential at the position of the MD) for the 3.5 and $6 \mathrm{~nm}$ thick films, with $\langle S\rangle=25$ and $19 \mathrm{~nm}$, respectively. For the $3.5 \mathrm{~nm}$ thick film, the fluctuation draws two minima at each side, $\sim 4 \mathrm{~nm}$ away from the MDs, where the chemical potential is reduced by $17 \mathrm{meV}$. This abrupt gradient in $\Delta \mu_{d}(x)$ should induce the preferential nucleation of LSMO at each side of the MDs, leading to the formation of the observed topographic pattern. It should be noted, however, that since in this case the glide plane is parallel to the interface plane, MDs can easily move to rearrange their positions and, therefore, those surface features do not necessarily appear associated with them. It is to be noted that there exists a kinetic limitation for the vertical growth of the ridges: Once a ridge is formed, the rapid increase in surface curvature at that point causes the competition between the surface energy, $\gamma \kappa(x)$, and strain energy, $w(x)$, terms of the chemical potential, eventually hindering its vertical growth. This scenario drastically changes as the film surface moves further apart from the dislocation strain sources (and those ones get closer), as exemplified by the $6 \mathrm{~nm}$ thick film (see Figure $4 \mathrm{~b}$ ). The plot clearly shows that a thickness increase of $2.5 \mathrm{~nm}$ results in nearly a $50 \%$ decrease in the amplitude of the fluctuation in $\Delta \mu_{d}(x)$. This effect contributes to homogenize the chemical potential throughout the surface, resulting in flatter films, in agreement with experimental observations (see Figure S1). Therefore, the formation of terraces in the $3.5 \mathrm{~nm}$ thick film, exhibiting a wider dispersion in $S$ values, can be attributed to the overlapping of the strain field in regions with locally enhanced MD densities.

\subsection{Strain effects on surface currents}


Before considering the local effect of buried MDs on surface currents, we will take into consideration the bulk transport behavior of the films. Figure 5a depicts standard four-point electrical resistivity measurements as a function of the thickness of the films. As seen in the figure, room temperature resistivity values decrease as the film thickness, thereof the relaxation level as manifested by the variation in the $c / a$ lattice parameter ratio, increases. The temperature dependence of the resistivity of the different films, on the other hand, indicates bulk-like insulating behavior for the $2 \mathrm{~nm}$ and $3.5 \mathrm{~nm}$ thick films, and metallic behavior for larger thicknesses (6 and 14nm), see Figure S2. As far as the surface electrical properties of the films are concerned, however, current maps indicate local resistivity fluctuations that appear associated with the formation of MDs. Starting with the $3.5 \mathrm{~nm}$ thick film, Figure $5 \mathrm{~b}$ shows an AFM image corresponding to the $I(x, z)$ current map shown in Figure 5c. Despite its bulk insulating behavior, this film exhibits surface metallic behavior. Current enhancements are clearly seen as dark contrasts decorating surface steps, which are attributed to the extended tip-surface contact area along their ledges. Within the terraces, brighter lines of depressed current are also observed along the in-plane $<100>$ directions. Both, topographic and conduction images are correlated with the underlying MD network. For the $6 \mathrm{~nm}$ thick films, although the topographic ridge/terrace pattern is no longer present (Figure 5d and S1), the corresponding current map still exhibits current depressions along lines parallel to the inplane $\langle 100\rangle$ directions, as clearly seen in Figures $5 \mathrm{e}$ and $\mathrm{f}$.

The influence of strain on the magnetotransport properties $\mathrm{ABO}_{3}$ perovskite compounds is intimately correlated to tilt and distortion processes of the $\mathrm{MnO}_{6}$ octahedral framework. Strain affects magnetotransport properties by acting on different mechanisms at a microscopic level. First strain affects both $<\mathrm{Mn}-\mathrm{O}-\mathrm{Mn}>$ bond angle and the $\mathrm{Mn}-\mathrm{O}$ bond length, thus modifying the strength of the double exchange ferromagnetic (DEF) interactions. For the same reasons strain also affects antiferromagnetic (AF) superexchange interactions. In addition, strain may introduce an orbital bias since in-plane compressive or tensile strains may 
promote selective $d 3 z^{2}-r^{2}$ or $d x^{2}-y^{2}$ orbital occupancy, respectively. Therefore, as a first approximation, an elongation of the $\mathrm{Mn}-\mathrm{O}$ distances or a decrease of the $\langle\mathrm{Mn}-\mathrm{O}-\mathrm{Mn}\rangle$ bond angle would promote a reduction of the strength of DEF interactions and therefore, a reduction of the ferromagnetic Curie temperature, $T_{\mathrm{C}}$, and an increase of electrical resistivity. On the contrary, the reduction of $\mathrm{Mn}-\mathrm{O}$ distances or the straightening of the $\langle\mathrm{Mn}-\mathrm{O}-\mathrm{Mn}\rangle$ bond angle promotes the strengthening of DEF interactions and a reduction of resistivity. The observation of enhanced conductivity at (100)-type twin walls in LSMO/STO thin films, which are submitted to a severe compressive strain, indeed supports these arguments. ${ }^{[4]}$ Nevertheless, this scenario may be strongly affected by selective orbital occupancy and AF superexchange interactions. The balance between these competing effects is controlled by the ratio between perpendicular and in-plane lattice parameters $c / a$, reflecting the degree of tetragonal distortion of the structure. ${ }^{[46]}$ In such scenario, increasing $c / a>1.0$ works against the metallic ferromagnetic behavior and would promote an increment of the resistivity. Since according to our analysis above, the behavior of the present films is well described by the continuum isotropic elasticity theory, the influence of rigid octahedral tilting mechanisms can be safely neglected, which otherwise would manifest as noticeable anomalies in the behavior of lattice parameters. ${ }^{[7]}$ Accordingly, the image contrast in the AFM current maps may be analyzed in terms of local $c / a$ values, directly governed by the residual misfit strain, $\left(\varepsilon_{x x}+\varepsilon_{0}\right)(x)$ shown in Figure 4a. The lateral modulation of the $c / a$ ratio at the film surface is given by $c / a(x)=\left(\varepsilon_{z z}(x)+1\right) /\left[\left(\varepsilon_{x x}+\varepsilon_{0}\right)(x)+1\right]$, where $\varepsilon_{x x}$ is given by Equation 2 , and $\varepsilon_{z z}$ is solely determined by the outwards relaxation determined by the Poisson's effect, $\varepsilon_{z z}=2 v \varepsilon_{x x} /(v-1)$. In the case of the $6 \mathrm{~nm}$ thick film, using the Poisson's ratio derived for the $2 \mathrm{~nm}$ thick film, $v=$ 0.35 (see above), a $c / a$ ratio of $\sim 1.03$ can be estimated at the locus of the dislocation $\left(\varepsilon_{x x} \sim-\right.$ 0.013 ) while $c / a \sim 1.0$ in a large area between dislocations $\left(\varepsilon_{x x} \sim 0\right)$. As a result, the metallic character is expected to be depressed at the dislocations that would exhibit a higher resistivity. 
Thus, the pattern observed in current maps is a close mimic of the dislocations pattern. The very same reasoning may be applied to explain the low conducting paths observed on the surface of the $3.5 \mathrm{~nm}$ thick film. In this case we find $c / a=1.05\left(\varepsilon_{x x} \sim-0.020\right)$ above the dislocation lines. Experimental determinations of the $c / a$ ratio from the GPA derived strain maps indeed support the above behavior. Figure 6a shows the $c / a(x)$ dependence at two different levels above the dislocation analyzed in Figures $1 \mathrm{c}$ and d, corresponding to the $3.5 \mathrm{~nm}$ thick film. The blue and red curves are taken close to the film surface and at half way from the buried dislocation core, as indicated in Figure 6b. It can be clearly observed that the $c / a$ ratio locally increases above the dislocation, reaching values closely similar to those derived from linear elasticity. It can be also observed that the average $c / a$ level increases towards the film surface, which can be attributed to an elastic relaxation perpendicular to the film surface.

Identifying the strained regions as less conducting is also in agreement with the insulating behavior observed in the $2 \mathrm{~nm}$ thick film, prior to the appearance of dislocations. In those films, $c / a \sim 1.06\left(\varepsilon_{x x} \sim-0.023\right)$, i.e., they are expected to lie well within an antiferromagnetic insulating phase. ${ }^{[6]}$ Above the onset of plastic relaxation, this analysis yields a view of partly relaxed LSMO/LAO thin films as a conducting compressed matrix, decorated with nanometric paths of higher resistance material aligned with the in-plane $<100>$ directions coinciding with the positions of the buried misfit dislocations, in agreement with experimental $I(x, y)$ maps. As shown above, the topological distribution of strained, high resistivity regions, throughout the volume of the films varies with the film thickness. Just after the onset of plastic relaxation, the MD density is low, but their associated strains occupy a significant fraction of the film volume owing to its reduced thickness. Thus, in the $3.5 \mathrm{~nm}$ thick film, the average bulk insulating behavior can be understood by considering that the connectivity between metallic regions is below the percolating threshold. At larger thicknesses, the 
increased dislocation density promotes a higher average level of relaxation consistent with the metallic behavior observed in the $6 \mathrm{~nm}$ thick film.

\section{Conclusion}

To conclude, the strain field of MDs in a complex oxide heterostructure introduces a lateral modulation of the chemical potential and the bandwidth dependent properties at the free surface of the film. In particular, the present experiments shed light on the structural mechanisms underpinning the topographic and electrical conduction patterning of the surface of LSMO/LAO thin films. It is shown that, even if perovskite thin films may relax misfit strains by combining octahedral distortions and octahedral tilting which may eventually elude the formation of MDs, the strain state of dislocated films is well described by continuum elasticity. This allows straightforward modeling of the strain sate of the films and their free surfaces, and the interpretation of their effect on local surface properties like the chemical potential and the bandwidth controlling the transport properties of these materials. This properties exhibit different dependencies on the amplitude of surface strain fluctuations, which in turn depend on film thickness and MD density. Chemical potential fluctuations are rapidly smeared out, limiting the thickness range useful for topographic patterning. On the other hand, surface current patterning is shown to persist up to larger thicknesses. Interestingly, our analysis indicates that current depressions along the projection of dislocation lines on the film surface are well described by the lateral modulation of the $c / a$ axial ratio, controlling the balance between selective Mn $d$ orbital occupation, in-plane Mn $3 d$

- O $2 p$ orbital overlapping, and AF interactions. ${ }^{[46]}$ These results demonstrate the feasibility of using MDs in a controlled way to produce spontaneous, highly ordered, surface topographic and conduction patterns on a $\sim 20 \mathrm{~nm}$ length scale. Further optimization of each of the surface 
properties investigated in this work can be achieved through the magnitude of the misfit strain and the strength of the Burgers vector of the MDs.

\section{Experimental section}

Films: High quality films with (100) orientation and thicknesses of 2, 3.5, 6 and 14nm, were epitaxially grown under a biaxial compressive strain of $\varepsilon_{0}=-2.3 \%$ on LAO substrates by magnetron sputtering as reported elsewhere. ${ }^{[47]}$

Electrical resistivity: The in-plane electrical resistivity was measured using the standard fourpoint geometry with a constant applied current of $5 \mathrm{nA}$.

Conducting Atomic Force Microscopy: Local electrical conductivity maps were measured by conducting atomic force microscopy (C-AFM) under a $\mathrm{N}_{2}$ environment, using commercial conductive $\mathrm{CrPt}$ coated Si tips mounted on cantilevers with $k=40 \mathrm{Nm}^{-1}$ (Budgetsensors). The lateral resolution of the technique is, in principle, limited by the tip radius of $\sim 10-20 \mathrm{~nm}$. In the employed set-up, the sample was grounded and the voltage was applied to the tip. An external I-V converter (Stanford Research Systems) was used to provide access to a wide range of compliance currents (1pA to $1 \mathrm{~mA})$.

High Resolution X-Ray Diffraction: The lattice parameters of the films were determined from high resolution $\mathrm{x}$-ray diffraction (HR-DRX) using a four-angle goniometer and primary optics consisting of a parabolic mirror and a 4 x Ge(220) asymmetric monochromator (X'Pert Pro MRD-Panalytical). The in-plane lattice parameters were determined in the same equipment with a parabolic mirror in the incidence beam optics, fixed grazing angle of $0.5^{\circ}$ on the sample, and parallel plate collimator in the diffracted optics.

Transmission Electron Microscopy: Thin foil specimens were prepared by conventional cutting-gluing-grinding procedures, followed by Ar milling at a grazing incidence down to perforation. Cross-section atomic resolution images (HRTEM) were obtained at 200kV using 
the field-emission gun FEI Tecnai F20 S/TEM and the Cs-corrected Tecnai F20 electron microscopes. The projected strain distribution on the plane of the film was directly imaged by low angle annular dark field (LAADF) microscopy, ${ }^{[48]}$ using a field emission gun Tecnai F20 S/TEM electron microscope.

Orientation Contrast Scanning Electron Microscopy: Orientation contrast SEM images, ${ }^{[49]}$ were obtained in a QUANTA FEI 200 FEG-ESEM electron microscope in order to determine dislocation densities over large areas.

\section{Acknowledgements}

We thank Dr. Belén Ballesteros for her assistance with electron microscopy experiments. This research was sponsored by the Spanish MINECO (MAT2011-29081-C02, MAT2012-33207 and MAT2013-47869-C4-1-P) projects. and the European Union Horizon 2020 research and innovation programme under the Marie Sklodowska-Curie grant agreement No. 645658. We also acknowledge financial aid from the Generalitat de Catalunya (2014 SGR 501). N. B. and F. S. also acknowledge funding from the European Union Seventh Framework Programme under Grant Agreement 312483-ESTEEM2 (Integrated Infrastructure Initiative I3) for providing access to aberration corrected electron microscope at CEMES (Toulouse). N. B. thank the Spanish MINECO for financial support through the FPI program. F.S.

acknowledges support from the Labex (Excellence Laboratory) NEXT for a visiting scientist fellowship at CEMES (Toulouse, France). Z.K. is grateful for the support from the Ministry of Education, Science, and Technological Development of the Republic of Serbia through Project III45018.

[1] H. Y. Hwang, Y. Iwasa, M. Kawasaki, B. Keimer, N. Nagaosa, Y. Tokura, Nat.

Mater. 2012, 11, 103. 
[2] P. Zubko, S. Gariglio, M. Gabay, Ph. Ghosez, and J. J.-M. Triscone, Annu. Rev. Cond. Mat. Phys. 2011, 2, 141.

[3] C. W. Bark, D. A. Felker, Y. Wang, Y. Zhang, H. W. Jang, C. M. Folkman, J. W. Park, S. H. Baek, H. Zhou, D. D. Fong, X. Q. Pan, E. Y. Tsymbal, M. S. Rzchowski, and C. B. Eom, Proc. Natl. Acad. Sci. USA 2011, 108, 4720.

[4] H. W. Jang, D. A. Felker, C. W. Bark, Y. Wang, M. K. Niranjan, C. T. Nelson, Y. Zhang, D. Su, C. M. Folkman, S. H. Baek, S. Lee, K. Janicka, Y. Zhu, X. Q. Pan, D. D. Fong, E. Y. Tsymbal, M. S. Rzchowski, and C. B. Eom, Science 2011, 331, 886.

[5] A. J. Millis, T. Darling, and A. Migliori, J. Appl. Phys. 1998, 83, 1588.

[6] A. Mukherjee, W. S. Cole, P. Woodward, M. Randeria, N. Trivedi, Phys. Rev. Lett. 2013, 110, 157201.

[7] F. Sandiumenge, J. Santiso, Ll. Balcells, Z. Konstantinovic, J. Roqueta, A. Pomar, J. P. Espinós, B. Martínez, Phys. Rev. Lett. 2013, 110, 107206.

[8] R. Ramesh and N. A. Spaldin, Nat. Mater. 2007, 6, 21.

[9] J. H. Haeni, P. Irvin, W. Chang, R. Uecker, P. Reiche, Y. L. Li, S. Choudhury, W. Tian, M. E. Hawley, B. Craigo, A. K. Tagantsev, X. Q. Pan, S. K. Streiffer, L. Q. Chen, S. W. Kirchoefer, J. Levy \& D. G. Schlom, Nature 2004, 430, 758.

[10] V. A. Shchukin and D. Bimerg, Rev. Mod. Phys. 1999, 71, 1125.

[11] D. J. Srolovitz, Acta Metallogr. 1989, 37, 621.

[12] S. C. Jain, A. H. Harker, and R. A. Cowley, Philos. Mag. A 1997, 75, 1461.

[13] S. Y. Shiryaev, F. Jensen, J. L. Hansen, J. W. Petersen, and A. N. Larsen, Phys. Rev. Lett. 1997, 78, 503.

[14] K. Häusler, K. Eberl, F. Noll, and A.Trampert, Phys. Rev. B 1996, 54, 4913.

[15] Y.-W. Lee and B. M. Clemens, Phys. Rev. B 2005, 71, 245416.

[16] H. Brune, M. Giovannini, K. Bromann, and K. Kern, Nature 1998, 394, 451. 
[17] I. Zeljkovic, D. Walkup, B. A. Assaf, K. L. Scipioni, R. Sankar, F. Chou, and V. Madhavan, Nat. Nanotech. 2015, 10, 849.

[18] M. Kuzmina, M. Herbig, D. Ponge, S. Sandlöbes, and D. Raabe, Science 2015, 349, 1080.

[19] D. Marrocchelli, L. Sun, and B. Yildiz, J. Am. Chem. Soc. 2015, 137, 4735.

[20] C. L. Jia, A. Thust, and K. Urban, Phys. Rev. Lett. 2005, 95, 225506.

[21] M. Kim, G. Duscher, N. D. Browning, K. Sohlberg, S. T. Pantelides, and S. J. Pennycook, Phys. Rev. Lett. 2001, 86, 4056.

[22] K. Szot, W. Speier, G. Bihlmayer, and R. Wasser, Nat. Mater. 2006, 5, 312.

[23] B. Dam, J. M. Huijbregtse, F. C. Klaassen, R. C. F. van der Geest, G. Doornbos, J. H. Rector, A. M. Testa, S. Freisem, J. C. Martinez, B. Stäuble-Pümpin, and R. Griessen, Nature 1999, 399, 439.

[24] F. Sandiumenge, T. Puig, J. Rabier, J. Plain, and X. Obradors, Adv. Mater. 2000, 12, 375.

[25] J. Suntivich, K. J.May, H. A. Gasteiger, J. B. Goodenough, and Y. A. Shao-Horn, Science 2011, 334, 1383.

[26] a) Z. Konstantinović, J. Santiso, Ll. Balcells, and B. Martínez, Small 2009, 5, 265; b) Z. Konstatinovic, F. Sandiumenge, J. Santiso, Ll. Balcells, and B. Martinez, Nanoscale 2013, 5, 1001.

[27] D. Ruzmetov, Y. Seo, L. J. Belenky, D.-M. Kim, X. Ke, H. Sun,V. Chandrasekhar, C.B. Eom, M. S. Rzchowski, and X. Pan. Adv. Mater. 2005, 17, 2869.

[28] M.-W. Chu, I. Szafraniak, R. Scholz, C. Harnagea, D. Hesse, M. Alexe and U. Gösele, Nat. Mater. 2004, 3, 87.

[29] C. L. Jia, S. B. Mi, K. Urban, I. Vrejoiu, M. Alexe, and D. Hesse, Phys. Rev. Lett. 2009, 102, 117601. 
[30] M. Arredondo, Q. M. Ramasse, M. Weyland, R. Mahjoub, I. Vrejoiu, D. Hesse, N. D. Browning, M. Alexe, P. Munroe, and V. Nagarajan, Adv. Mater. 2010, 22, 2430.

[31] A. Lubk, M. D. Rossell, J. Seidel, Y. H. Chu, R. Ramesh, M. J. Hytch, and E. Snoeck, Nano Lett. 2013, 13, 1410.

[32] A. Urushibara, Y. Morimoto, T. Arima, A. Asamitsu, G. Kido, Y. Tokura, Phys. Rev. $B$ 1995, 51, 14103.

[33] A. Bernand-Mantel, P. Seneor, K. Bouzehouane, S. Fusil, C. Deranlot, F. Petroff, and A. Fert, Nat. Phys. 2009, 5, 920.

[34] B. D. Terris and T. Thomson, J. Phys. D: Appl. Phys. 2005, 38, R199.

[35] M. -C. Daniel and D. Astruc, Chem. Rev. 2003, 104, 293.

[36] Z. Wang, M. Saito, K. P. McKenna, and Y. Ikuhara, Nat. Comms. 2014, 5, 3239.

[37] M. J. Hÿtch, Microsc. Microanal. Microstruct. 1997, 8, 41.

[38] R. Hull and J. C. Bean, Crit. Revs. Sol. St. Mater. Sci. 1992, 17, 507.

[39] J. P. Hirth and J. Lothe, Theory of Dislocations, McGraw-Hill, New York, USA.

[40] T. W. Darling, A. Migliori, E. G. Moshopoulou, S. A. Trugman, J. J. Neumeier, J. L. Sarrao, A. R. Bishop, and D. J. Thompson, Phys. Rev. B 1998, 57, 5093.

[41] X. Luo and B. Wang, J. Appl. Phys. 2008, 104, 073518.

[42] X. Wu and G. C. Weatherly, Semicond. Sci. Technol. 2003, 18, 307.

[43] G. Springholz, Appl. Surf. Sci. 1997, 112, 12.

[44] G. Springholz, G. Bauer, and V. Holy, Phys. Rev. B 1996, 54, 4500.

[45] Ll. Balcells, M. Paradinas, N. Bagués, N. Domingo, R. Moreno, R. Galceran, M. Walls, J. Santiso, Z. Konstantinovic, A. Pomar, M.-J. Casanove, C. Ocal, B. Martínez, and F. Sandiumenge, Phys. Rev. B 2015, 92, 075111.

[46] Z. Fang, I. V. Solovyev, and K. Terakura, Phys. Rev. Lett. 2000, 84, 3169.

[47] Z. Konstantinovic, J. Santiso, D. Colson, A. Forget, Ll. Balcells, B. Martínez, J. Appl. Phys. 2009, 105, 063919. 
[48] L. Fitting, S. Thiel, A. Schmehl, J. Mannhart, and D. A. Muller, Ultramicroscopy 2006, $106,1053$.

[49] D. J. Prior, A. P. Boyle, F. Brenker, M. C. Cheadle, A. Day, G. Lopez, L. Peruzzo, G. J. Potts, S. Reddy, R. Spiess, N. E. Timms, P. Trimby, J. Wheeler, L. Zetterström, Am. Miner. 1999, 84, 1741. 


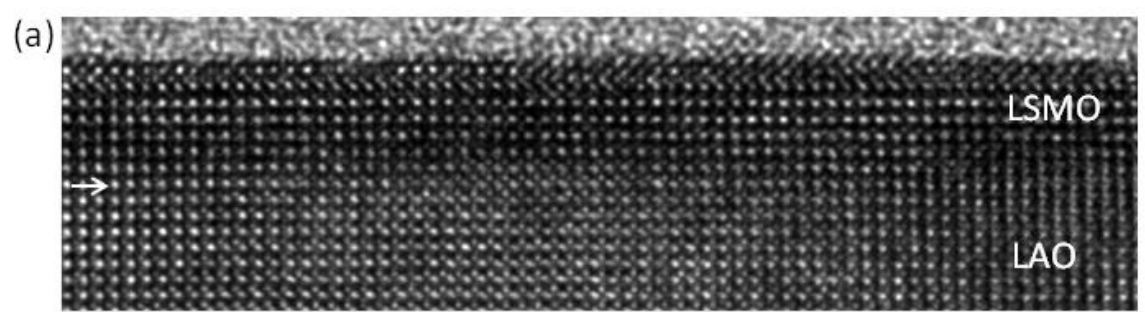

(b)

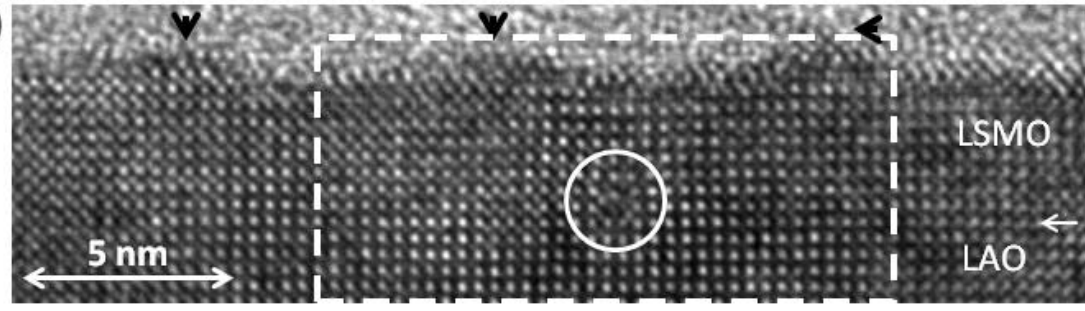

(c)
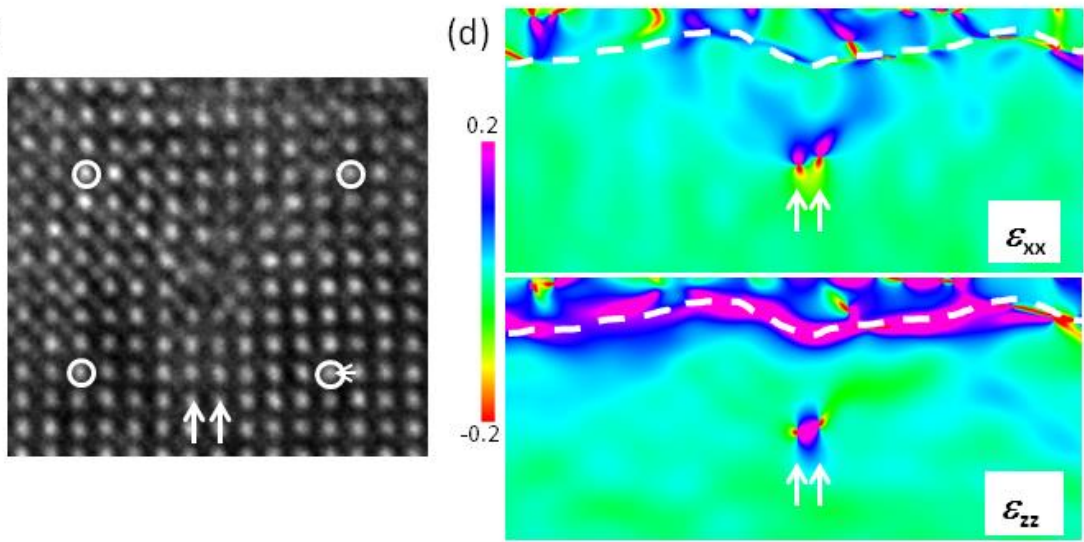

Figure 1. a) Cross-section HRTEM image of a 2nm thick LSMO/LAO film, exhibiting a coherent, fully strained, interface (indicated by an horizontal arrow), and a flat surface. $b$ ) Cross-section HRTEM image corresponding to a $3.5 \mathrm{~nm}$ thick film, exhibiting a dislocated interface (a MD is shown inside the white circle) and an undulated surface (outgrowths are indicated by black arrowheads); the boxed area corresponds to the strain maps shown in d). c) Magnified view of the dislocation core encircled in b); white circles mark the corners of a Burgers circuit yielding Burgers vector, $\mathbf{b}_{\mathrm{x}}=a_{\mathrm{LAO}}[100]$; the two vertical arrows indicate two extra half-planes consistent with a split core consisting of two 1/2[100] partials; careful inspection of the image reveals a downwards bending of atomic rows above the core. d) Inplane $\left(\varepsilon_{\mathrm{xx}}\right)$ and out-of-plane $\left(\varepsilon_{\mathrm{zz}}\right)$ strain maps, corresponding to the rectangular dashed window shown in $b$ ), as determined from GPA analysis; the two vertical arrows indicate the positions of the two partials building the split core; the surface of the film is indicated by a dashed line; the color scale bar corresponds to a full strain range of \pm 0.2 (negative and positive being compressive and tensile, respectively). 

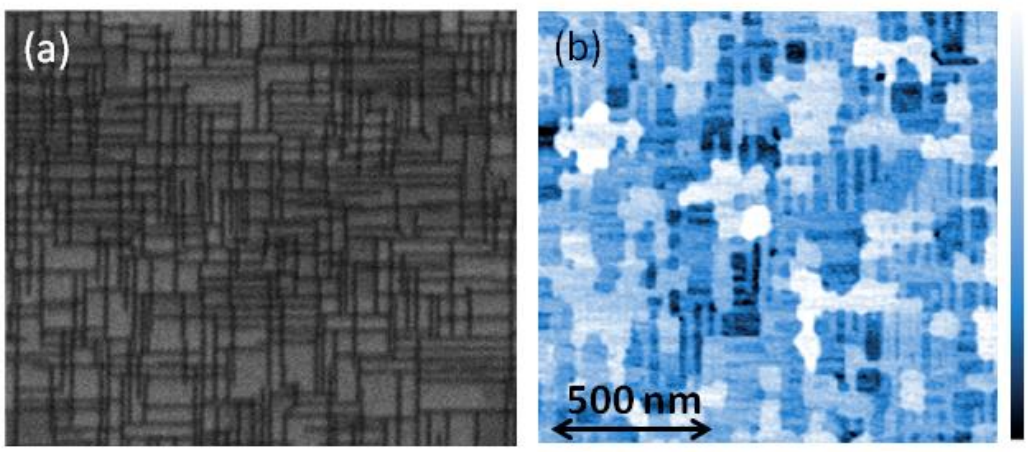

Figure 2. a) AFM image showing the topography of a 3.5nm thick film, consisting of ridges aligned with the [100] and [010] directions, and terraces; the color scale bar corresponds to a full range of $1.5 \mathrm{~nm}$. b) Orientation contrast SEM images revealing the MD network of the same film. The scale bar is the same for both images. It can be observed that the ridge pattern mimics the underlying buried dislocation network. 

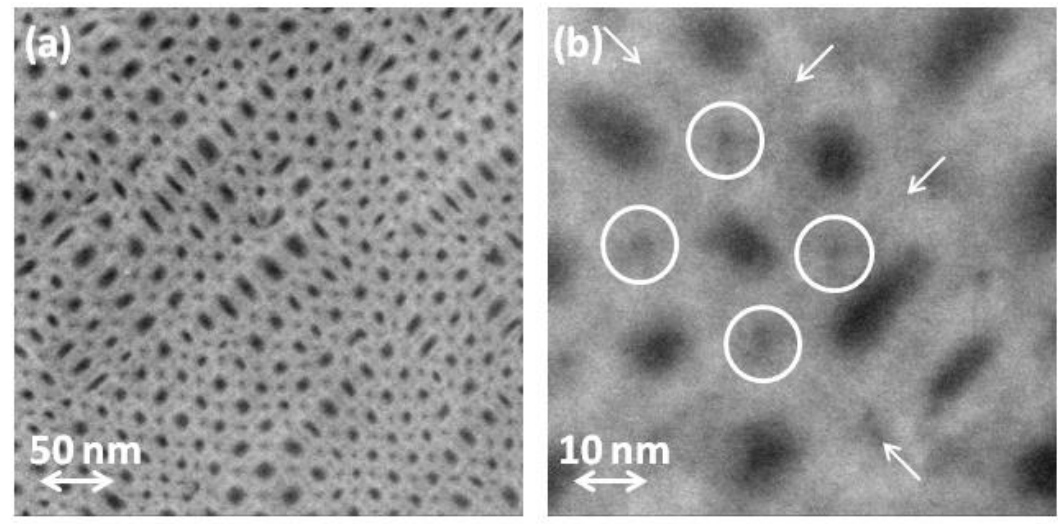

(c)

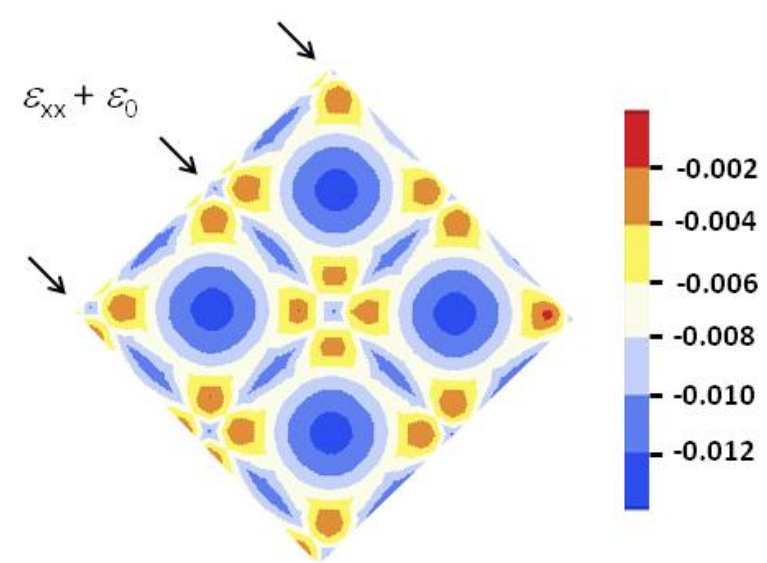

Figure 3. a) Planar view strain sensitive LAADF image corresponding to a $6 \mathrm{~nm}$ thick film. b) Enlarged view of a) showing in detail the strain contrast associated with the buried MD network; arrows indicate dislocation lines running along the [100] and [010] directions, and circles mark their intersections. c) Calculated distribution of residual strains $\left(\varepsilon_{x x}+\varepsilon_{0}\right)(x, y)$ in a similar film; arrows indicate dislocation lines, and colors correspond to different strain levels, as indicated in the color scale bar. 
(a)

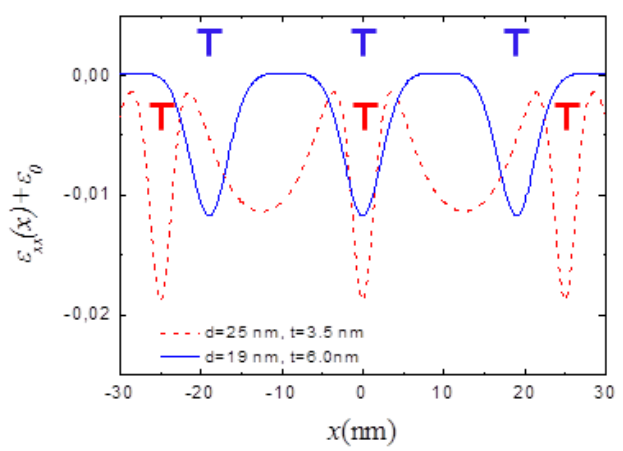

(b)

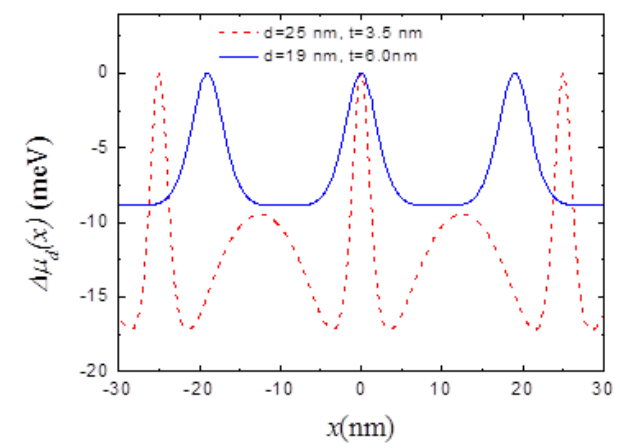

Figure 4. a) Calculated modulation of in-plane strain at the surface of 3.5 and $6 \mathrm{~nm}$ thick films, originated from buried MDs; the spacing between MDs is the average spacing determined experimentally for each thickness. b) Corresponding modulation of the surface chemical potential. The location of dislocations for each thickness is indicated in a). 

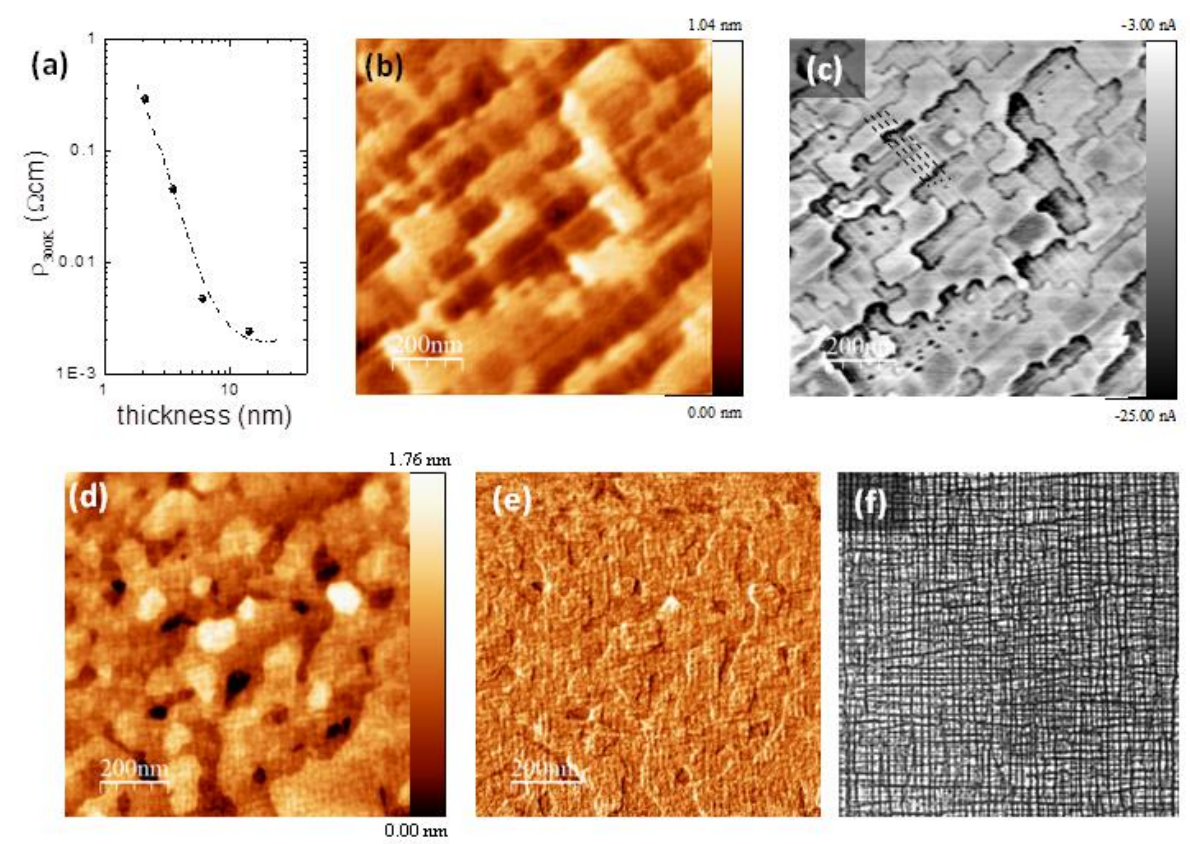

Figure 5. a) Room temperature thickness dependence of the resistivity of the LSMO/LAO films. b) Topography and c) simultaneous current map, of a $3.5 \mathrm{~nm}$ thin film. Dashed lines on the current map serve as a guide to the eye to highlight the dislocation network at the surface. d) Topography and e) the corresponding normal force, of a $6 \mathrm{~nm}$ thick film. f) Handmade drawing to emphasize the dislocation network. 


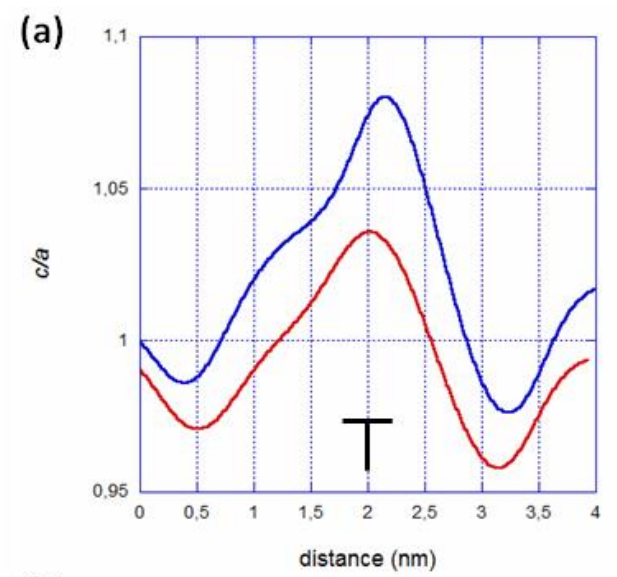

(b)

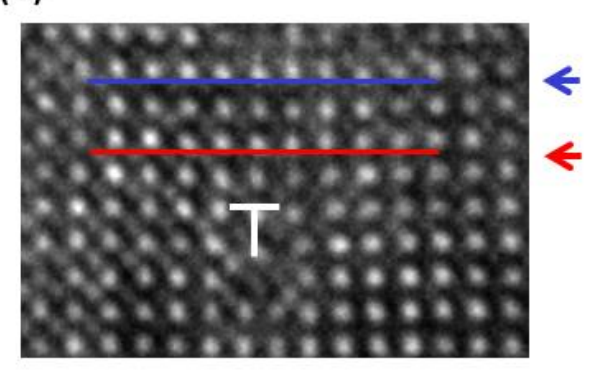

Figure 6. a) Variation of the $c / a$ ratio across the position of a buried MD as determined from the experimental $\varepsilon_{x x}$ and $\varepsilon_{z z}$ strain maps shown in Figure 1d. b) Selected area of the HRTEM image shown in Figure 1d, showing the positions of the profiles shown in a); the red and blue lines correspond to the red and blue curves, respectively. 


\section{Toc entry}

Misfit dislocations in $\mathrm{La}_{0.7} \mathrm{Sr}_{0.3} \mathrm{MnO}_{3} / \mathrm{LaAlO}_{3}$ compressively strained heterostructures are shown to induce topographic and surface current patterning. The underpinning mechanism of this behavior is a lateral modulation of surface strain influencing the chemical potential $(\Delta \mu)$ and the bandwidth $(W)$ at the free surface, providing a clue for the realization of spontaneous functional nanostructures in the $\sim 20 \mathrm{~nm}$ range.

complex oxides, misfit dislocations, strain, surface patterning, surface current

Felip Sandiumenge,* Núria Bagués, José Santiso, Markos Paradinas, Alberto Pomar, Zorica Konstantinovic, Carmen Ocal, Lluís Balcells, Marie-Jo Casanove, and Benjamín Martínez

Misfit Dislocation Guided Topographic and Conduction Patterning in Complex Oxide Epitaxial Thin Films

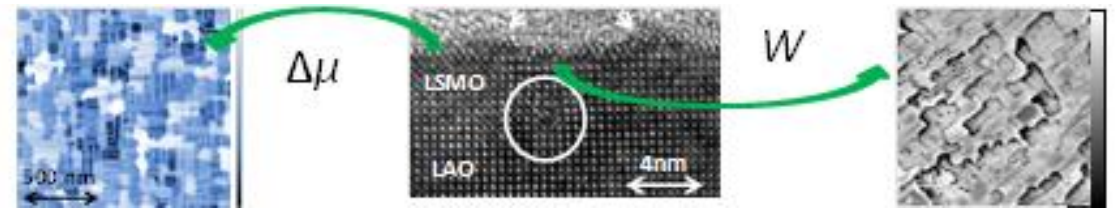




\section{Supporting Information}

\section{Misfit Dislocation Guided Topographic and Conduction Patterning in Complex Oxide Epitaxial Thin Films}

Felip Sandiumenge, * Núria Bagués, José Santiso, Markos Paradinas, Alberto Pomar, Zorica Konstantinovic, Carmen Ocal, Lluís Balcells, Marie-Jo Casanove, and Benjamín Martínez

(a)
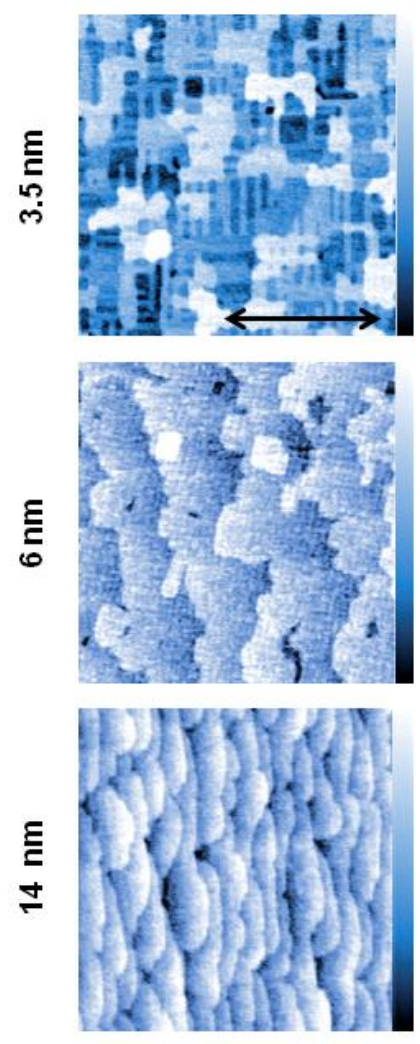

(b)
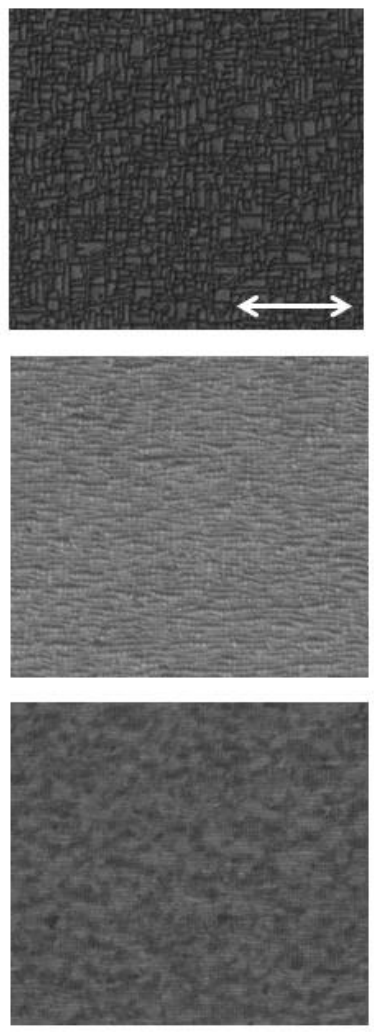

(c)
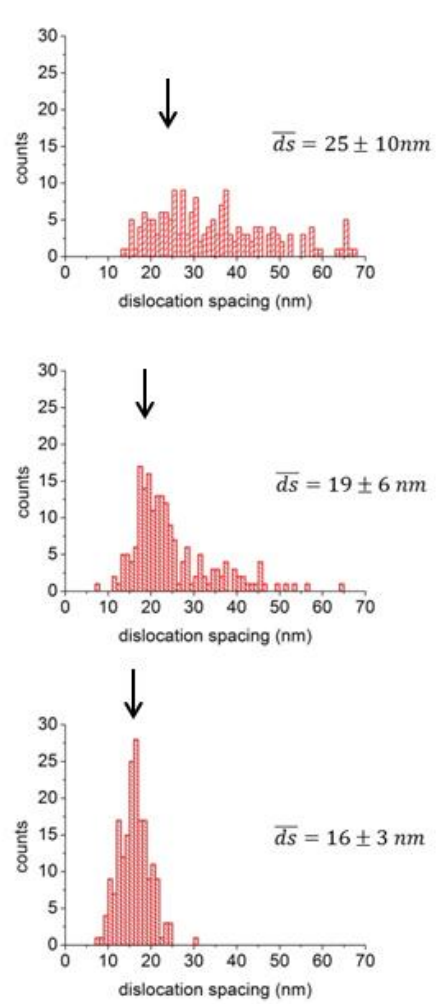

Figure S1. a) Topographic AFM images, b) OC-SEM images revealing the misfit dislocation network, and c) histograms of dislocation spacings, corresponding to 3.5, 6 and $14 \mathrm{~nm}$ thick films. Scale bars are common for AFM and OC-SEM images and correspond to 500nm. 

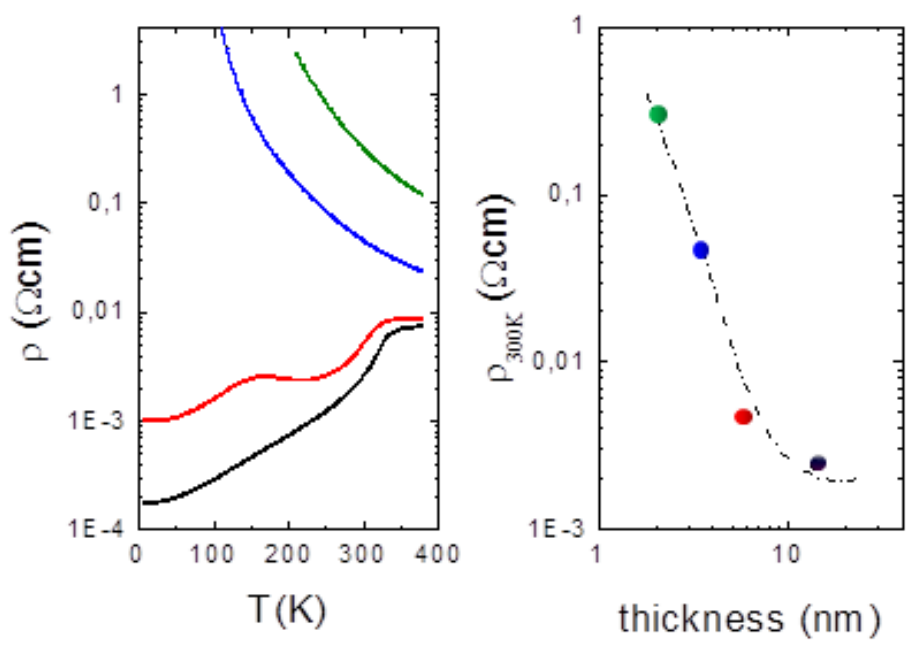

Figure S2. Left panel shows the temperature dependence of the resistivity for 2, 3.5, 6 and $14 \mathrm{~nm}$ thick films. Corresponding resistivity values at $300 \mathrm{~K}$ are reminded in the right panel. 